\title{
Werner Cee
}

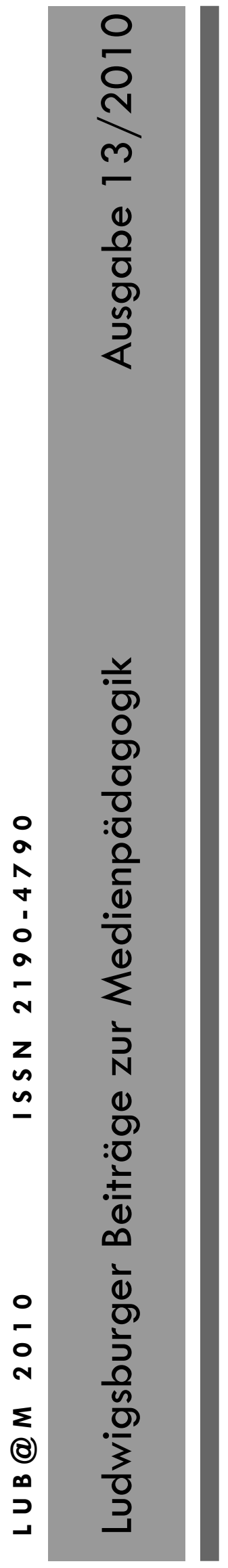

Die Veranstalter „KulturRegion Stuttgart e.V.“ und „Musik der Jahrhunderte Stuttgart“ mit dem „Netzwerk Süd“ organisierten vom 1. bis 10. Oktober 2010 das Festival „Zukunftsmusik“. Zwölf Komponisten haben musikalische Projekte für 12 Städte der KulturRegion Stuttgart entworfen und dabei besondere Merkmale des jeweiligen Ortes hervorgehoben. Als Beitrag der Stadt Ludwigsburg zum diesjährigen Projekt konnte eine Zusammenarbeit zwischen der Pädagogischen Hochschule Ludwigsburg und dem Komponisten Werner Cee realisiert werden. In einer Preview und als Vorgeschmack auf die endgültige Produktion zeigten der Komponist und Musik- und Kunststudierende der PH Ludwigsburg im PH-Innenhof am 22. Juli in einer Live-Performance das multimediale Klangportrait „Alltags(Ver-)dichtung“. Im Anschluss daran sprach Prof. Dr. Peter Imort mit dem Komponisten und Medienkünstler Werner Cee über die Aufführung der $\mathrm{Zu}-$ kunftsmusik.

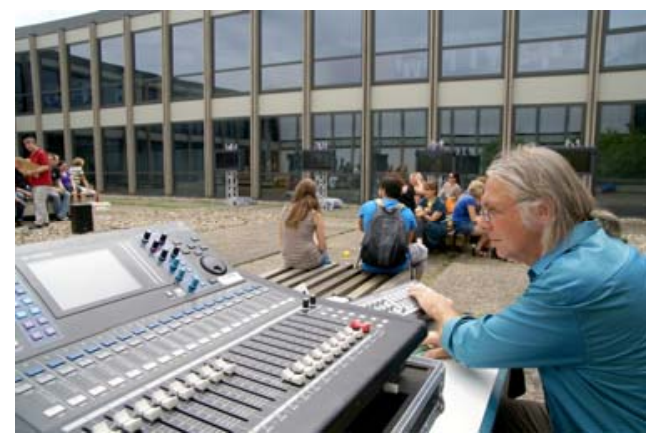

\section{Zur Person}

Studium der Malerei an der Hochschule für Bildende Künste in Frankfurt/M.; ebenso intensive Beschäftigung mit Musik, auch auf experimenteller Basis. Arbeiten im Bereich von Medienkunst mit Schwerpunkt auf elektroakustischen Kompositionen und ArsAcustica-Produktionen. Zahlreiche Dokumentationen, Soundscapes sowie musikethnologische Features für den Rundfunk (u.a. für WDR, HR, SWR, BR und für DRadio Kultur). Unterrichtstätigkeit an der Kunsthochschule Saarbrücken, an der Hochschule der Künste in Bern, an der PH Ludwigsburg. Bedeutende Preise und Auszeichnungen, zuletzt 2010 der „Prix Italia“ für die Radiokomposition „Winterreise featuring Schuberts Winterreise“. Homepage:

www.wernercee.de/wernercee/home.html
Imort: Die erste Aufführung der Zukunftsmusik Ludwigsburg liegt hinter dir. Was sind deine Eindrücke vom Tag gewesen?

Cee: Erst einmal hat mich gefreut, dass alles so prima geklappt hat. Viele der Studierenden haben sich auf das Projekt eingelassen und bekamen eine Idee davon, was möglich sein kann. Mit der heutigen Aufführung gab es das erste Mal Gelegenheit zu spüren, was für eine Faszination und Überraschung darin steckt, wenn eine professionelle Anlage dahintersteht. Das finde ich gut, das ist gelungen.

Imort: Arbeitest du bäufiger mit Studierenden zusammen?

Cee: Ich habe ein Jahr lang unterrichtet, von daher habe ich einige Erfahrung mit Studierenden. Es ist natürlich ein Unterschied, ob es angehende Musikpädagogen/Musikvermittler oder Komponisten sind. Beispielsweise sehe ich Unterschiede in der Herangehensweise. Komponisten, die das ganze Leben voll auf diese Schiene setzen, liefern sich solchen Sachen anders aus und wissen um die enge Gratwanderung, denn was man hier tut, ist mit Unsicherheiten verbunden. Man geht ein Risiko ein und denkt weniger abgesichert. In der Aufführung weiß man, das ist jetzt diese eine Chance - die unter Umständen selten ist - und man versucht, das nächste Mal noch besser zu werden. Unter diesen Bedingungen wird schärfer an die Sache herangegangen, als wenn man eher das Spielerische daran sieht. Dennoch gab es Ähnlichkeiten im ästhetischen Ansatz. Bei den Studierenden, die in den Produktionen fest mitgearbeitet haben, war klar, dass die sich einiges an Videokunst angeguckt hatten, das habe ich gemerkt.

Imort: Konntest du in den Monaten, in denen du mit den PH-Studierenden gearbeitet hast, eine Entwicklung erkennen, vielleicht auch in der ästhetischen Einstellung?

Cee: Am Anfang hatte ich das Gefühl, dass einige ein bisschen von den härteren Klangbeispielen, die ich mitgebracht hatte, geschockt waren. Die anfängliche Scheu hat sich dann gelegt, das war deutlich. Ich glaube schon, dass die eigene Praxis einiges bewirkt hat, als es an die Geräte ging und sie selbst loslegen konnten. 
Wenn man eine Sache gut ausführt und man ein Forum hat, wo die Leute Vertrauen haben, dann lassen sie sich auch darauf ein. Deshalb ist es so wichtig, dass man beispielsweise eine wirklich gute Lautsprecheranlage zur Verfügung hat, die richtig gut gepegelt ist und die gut funktioniert. Sonst entsteht der Eindruck, dass alles nur Lärm und Krach ist. Aber wenn es wirklich sitzt, dann werden die meisten neugierig. Aber man darf nie Schmalspur machen für Menschen, die hier ästhetisches Neuland betreten, die hier unbedarft sind. Immer volle Pracht also, dann funktioniert es auch gut, das ist meine Erfahrung. Das betrifft im Übrigen auch die Aufführung selbst. Dann kommt auch der Hausmeister und redet eine halbe Stunde mit mir nach dem Konzert.

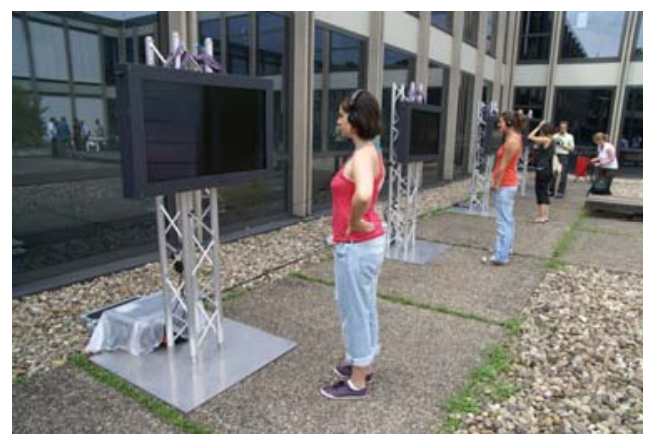

Imort: Also hast du ein vermittelndes Interesse mit dem, was du tust?

Cee: Ich will Publikum und ich will Austausch haben. Ich mache solche Projekte auch deshalb sehr gern, weil sie mich immer neu inspirieren. Ich erzähle auch bereitwillig, was ich gemacht habe, wie man etwas anpacken kann, und es interessiert mich, wie andere Leute, andere Altersgruppen darauf reagieren.

Imort: Du arbeitest als Komponist mit Neuen Medien. Hast du eine pädagogische Perspek.tive speziell auch für Jugendliche?

Cee: Was ich wichtig finde, ist, dass Kinder und Jugendliche ganz früh lernen, Medien lesen zu können. Ich denke da an Alltagsmedien wie Fernsehen, Radio und Internet. Die sind so alltäglich, dass man sie als gottgegeben hinnimmt. Dass aber hinter allen Medien Menschen stehen, die das bewusst so setzen, das nimmt man einfach so hin. Man macht den Fernseher an und es kommt etwas. Dabei wird vergessen, dass jemand absichtsvoll dieses Ding jeden Abend gestaltet. Zum Beispiel war das ganz deutlich während der Berichterstattung über den Irakkrieg zu sehen. Die Bilder waren häufig nachtgrün. Allerdings waren Nachtsichtgeräte gar nicht immer nötig, das spezielle Licht war nur ein Gestaltungsmittel. Und wie es dann hieß, der Krieg ist nun vorbei, gewonnen sozusagen, da wurden die Nachtsichtgeräte gewissermaßen ausgeschaltet und es waren wieder normale Lichtverhältnisse zu sehen. So wird die ganze Zeit mit mediengestalterischen Mitteln gearbeitet. Es ist eine ganz wichtige Sache, diese Mittel bewusst wahrzunehmen. Hier denke ich dann an Schule, an das, was Kinder dringend lernen sollten.

Imort: Hat das Dechiffrieren von Medien für dich immer einen kritischen Impetus?

Cee: Ja, für mich hat es das schon. Medien sind unglaublich potent als Mittel. Man muss aufpassen, immer auf dem Laufenden zu bleiben. Es gibt heute unglaublich gute und ausgefuchste Leute, die mit Medien arbeiten. Es ist nicht nur wie früher die so genannte manipulative Werbung, es ist inzwischen ein ganz anderes Level erreicht worden. Ich halte es für wichtig, das einschätzen, interpretieren und entziffern zu können.

Imort: Medien entziffern zu können ist die eine Seite, damit $z u$ arbeiten die andere?

Cee: Ich denke, man versteht eine Sache um so mehr, je intensiver man damit arbeitet. Hier durch die PH-Arbeit weiß ich nun, der Studierende macht das so, der andere macht das so und die macht das vielleicht etwas besser und der hat noch einen anderen Trick raus. Ich bin ohnehin überzeugt von diesem Lernen durch Tun. Gewiss, dazu gehören Hintergrundinformationen und Hintergrundwissen, aber letztendlich so richtig lernt man es durchs Machen. Das ist eine Einstellung fast noch wie aus einer alten Handwerkstradition, wie in einer künstlerischen Werkstatt.

Mit Handwerk meine ich nicht das Handling von Technik, das muss man in jedem Fall können, das ist die Grundvoraussetzung. Ich meine damit, den Klang einzuschätzen und zu erkennen, wie ich ihn einsetzen kann. Das musikalisch-handwerkliche Denken ist ebenso wichtig, die Ästhetik zu verstehen. Wichtig ist, ein richtig gutes Gespür zu entwickeln; wie ein Handwerker, der Holzstücke nimmt und sagt, dieses nehme ich für das Tischbein und das andere für die Zarge. So kann man auch mit Tonaufnahmen umgehen. Man weiß mit der Zeit dann ganz genau, wo eine Aufnahme ist, die etwas bringt, die außergewöhnlich ist. In diesem Sinne Handwerker zu sein bedeutet, das Klangmaterial zu beherrschen. Mit „beherrschen“ meine ich weniger das Computerprogramm, sondern das Klangmaterial selbst.

Imort: Bedingt dein Verständnis einer künstlerischen Werkstatt auch Instrumente?

Cee: Wenn man Klänge sucht und aufnimmt, sind der Lautsprecher und das Mikrofon die Instrumente. Wenn ich Töne und Klänge von einer Stadt, einem Ort aufnehme und übertrage, dann müssen sie klingen. 
Da ist nur die sinnliche, in dem Fall auditive Sensation ausschlaggebend. Es geht natürlich nicht um eine naturalistische Materialsammlung. Das Auto, das in der Stadt fährt, ist künstlerisch sinnlos. Erst durch die Übertragung in einen qualitativ anderen Kontext wird es möglich, dass ein Auto, das vorbeifährt, nicht nur als Auto, sondern als richtige Klang-Sensation wahrgenommen wird, was sehr expressiv sein kann. Dabei kann sich eine ganz starke musikalische Qualität entwickeln, obwohl die Strukturen nicht in engem Sinne musikalisch sind, aber von der Expressivität eben schon.

Die Klang-Sensation kann ein konkreter Klang, den ich im richtigen Moment in den Raum setze, genauso sein wie eine Posaune, in die ich blase. Das ist mir völlig egal. Ja gut, ich sitze dann an Reglern, während der Musiker mit seinem ganzen Körper hinter seinem Instrument steht und artikulieren muss. Aber so eine Tastatur ist schon ein richtiges Instrument, das man beherrschen muss. Beispielsweise hat das Pegeln der Regler, das Setzen von Punkten und die Entscheidung, wann ein Schnitt kommt schon die Kraft vom Instrumentenspiel. Ja, und ob ich nun einen konkreten Klang nehme und den spiele und möglicherweise mit Equalizer etc. beeinflusse oder ein traditionelles Instrument, ist letztlich egal. Ich muss keine Orgel spielen, ich kann auch Stadtklänge spielen, die letzten Endes auch wirken wie ein Synthesizer.

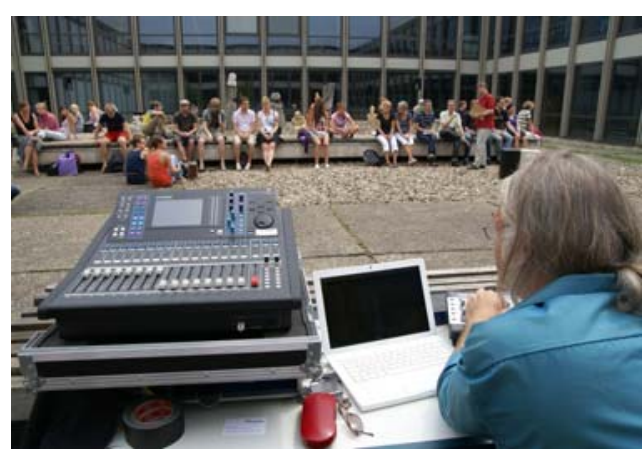

Imort: Du kommst ursprünglich von der Bildenden Kunst her. Ist es da nicht naheliegender, wenn du Videokünstler geworden wärst?

Cee: (Lacht.) Ich hab aber immer auch Musik gemacht. Und bin in der Schule schon aus dem Musikleistungskurs rausgeflogen, weil ich in Notenlehre schon immer leistungsschwach war und meine Sachen machen wollte, aber nie durfte.

Ich hab mir meinen eigenen Weg gesucht. Ich komme von der Rockmusik und vom Freejazz her und da haben wir immer mit Instrumenten experimentiert. Obwohl ich mehr von der Bildenden Kunst kam, habe ich mich mit meinen Soundgeschichten für ein Stipendium beworben. So kam es, dass ich ein Musikstipendium im Schloss Solitude erhielt. Ich weiß noch, wie der Juryvorsitzende mich anrief und mich zur Auswahl nach Köln eingeladen hat. Er hat gesagt, dass jedem Stipendiat ein Steinway-Flügel gestellt wird. Ich hatte damals solch einen Respekt vor den NeueMusik-Komponisten, dass ich von dem Moment an gedacht habe, ich hätte keine Chance mehr. Ich konnte mit einem Flügel nichts anfangen, doch dazu meinte er nur: „Schmieren sie ihr Butterbrot darauf." Von da an war das Eis gebrochen und wir haben geplaudert. Er hat versucht, eine Gruppe schräg zusammenzustellen.

Imort: Es ist noch nicht alliu lange möglich, Rockmusike oder experimentelle Musik in dieser Komplexität computergestützt zu machen. Wie ist die Entwicklung neuer Medientechnologien mit deiner künstlerischen Biografie zusammengestoßen?

Cee: Also, für die ersten Radioproduktionen wurde noch viel geschnipselt. Ich erinnere mich an den SWR, da wurden Leute aus dem Nachbarstudio gerufen, wenn fünf Bandmaschinen gleichzeitig gestartet werden mussten und alle die Knöpfe gedrückt hielten. Die Phase stand an meinem künstlerischen Anfang und sie war relativ bald passé. Zu Hause hat es länger gedauert. Es gab die ersten Sampler, die nur ein paar Sekunden aufnehmen konnten und Mehrspurbandgeräte, die man dann starten musste. Und irgendwann hatte ich dann Pro Tools in den Fingern, das ist vielleicht 13 oder 14 Jahre her.

Imort: Sind deine Live-Kompositionen dadurch komplexer geworden oder hast du vorher einfach mehr Arbeit investiert?

Cee: Wie mich selbst gab es natürlich schon vorher Leute, die sehr komplex gearbeitet haben. Ich denke an die Musique concrète oder die Elektronische Musik, die sind ja schon sechzig, fast siebzig Jahre alt. Ja, aber dass man Zugriff auf alles hat, was irgendwie klingt, das ist neu. Ebenso wie diese Massen an Material, die vielen Soundpools im Internet, wo sich viele bedienen. Hier bin ich ein großer Skeptiker, ich nehme immer nur die eigenen Aufnahmen.

Imort: Warum diese Skepsis?

Cee: Ich bin mir sicher, dass verschiedene Personen, denen ich das gleiche Mikrophon gebe und die die gleiche Klangszenerie aufnehmen, mit anders klingenden Aufnahmen zurück kommen. Der eine nimmt vielleicht nur ein separiertes Geräusch auf und ein anderer auch alles, was darum herum zu hören ist. Und schon werden zwei ganz unterschiedliche Richtungen eingeschlagen. Ich lasse sehr viele Zufälligkeiten zu und montiere sie wieder. Das ergibt wiederum ganz neue Effekte, indem beispielsweise das Hintergrundgeräusch zum Vordergrund und damit zum Wichtigen wird. Außerdem ist es ein handwerklicher Ergeiz von mir, aus einer Aufnahme das herauszukitzeln, was mit je speziellen Mikrophonen möglich ist. 
Imort: Die nächste Aufführung wird am 07.10.2010 in der Reithalle der Karlskaserne stattfinden. Was wird sich gegenüber der Juli-Auffübrung noch verändern?

Künstlerisch werde ich noch detaillierter an den Sachen arbeiten. Manches hatte noch zu sehr den Charakter einer Materialsammlung. Dazu werde ich die Fülle an Samples noch einmal genau durchhören, praktisch auswendig lernen. Das ist ganz, ganz viel Fleißarbeit. Außerdem ist mir aufgefallen, dass viele Samples ziemlich weich einsetzen. Eine Möglichkeit ist, sie anzuschneiden, um deutliche Einstiege zu setzen. Insgesamt werden die Choreografien und Räumlichkeiten von den Klängen und Sounds mehr ausgearbeitet. Vor allem wird dann das Zusammenspiel mit Eivind Aarset (Norwegen, git/electronics), Till Hillbrecht (Schweiz, turntables/electronics) und Tord Knudsen (Norwegen, visuals) spannend! Imort: Werner Cee, vielen Dank für das Gespräch. 